\title{
Option pricing and hedging bounds in incomplete markets
}

\author{
Tao Hao* \\ "Watson Wyatt Worldwide, London, UK. \\ Tel: + 441737 274503, Fax: + 441737 241496, E-mail: tao.hao@watsonwyatt.com, \\ http://www.watsonwyatt.com
}

Received (in revised form): 10th September, 2007

\begin{abstract}
Tao Hao is an economist within a global research team. His role is to develop research across a broad range of areas of strategic interests to Watson Wyatt, including issues concerning corporate pension risk exposure, stochastic financial modelling, asset allocation strategy, derivatives pricing and risk management, dynamic hedging strategy for pension scheme, asset and liability management. Tao holds BSc in Economics from Beijing University, China, and an MSc in Finance and Economics from London School of Economics, UK. He is currently undertaking his PhD study in Finance and Economics at Birkbeck College, University of London.
\end{abstract}

\section{Practical applications}

Black-Scholes option pricing model is widely used within the asset and risk management of traditional and alternative investments. From the investors' point of view, Black-Scholes model provides a unique price of a contingent claim in an ideal, complete and unconstrained market based on the fundamental principles of 'absence of arbitrage opportunities'. The assumptions of the model, however, may not be closely met in practice. I show various approaches to derive closed-form solutions of tight upper and lower bounds, and implications on delta hedging strategies.

\begin{abstract}
This paper has reviewed the literature on option pricing in incomplete markets. Tight upper and lower bounds can be derived based on the assumptions of mean and variance of the underlying asset price, not on its entire distribution. The differences between estimated upper or lower bounds and Black-Scholes price are quite small for deep in-the-money options, but can be very significant for deep out-of-the-money options. But at the same time, despite the wide pricing bounds, analysis of the implied hedging bounds suggests that the implications for asset allocation of incomplete markets are fairly limited.

Journal of Derivatives \& Hedge Funds (2008) 14, 78-89. doi:10.1057/jdhf.2008.9
\end{abstract}

Keywords: Black-Scholes; option pricing; upper and lower bounds; hedging; incomplete market

\section{INTRODUCTION}

The seminal Black-Scholes ${ }^{1}$ model provides the unique price of a contingent claim in an ideal, complete and unconstrained market based on the fundamental principles of 'absence of arbitrage opportunities'. In other words, this price is unique if there are no arbitrage opportunities by taking either a short or long position in the claim and investing wisely in the market. The Black-Scholes model also implies a
Journal of Derivatives \& Hedge Funds, Vol. 14 No. 2, 2008, pp. 78-89 (C) 2008 Palgrave Macmillan Ltd 1753-9641 
single hedging portfolio, which one can use to exactly duplicate the claim. The Black-Scholes model has been extended in a multitude of directions and, as is well known with complete markets, the results generalise and produce a unique hedging portfolio.

The assumptions of the model, however, may not be closely met in practice. This could be either because markets are incomplete or because there is underlying model error. Market incompleteness generally comes from two main sources:

1 There are not enough assets in the market to 'span' the uncertainty. (An example would be standard stochastic volatility.)

2 Trading strategies are limited or not ideal because of discrete trading or transaction costs, short selling constraints, etc.

This paper provides a synthetic review of the literature on these issues and the consequences for hedging portfolios. In incomplete markets, instead of a single arbitrage-free price there appears an 'arbitrage-free' interval $\left[h_{\text {low }}, h_{u p}\right]$, which contains the complete markets price. Here, $h_{u p}$ represents the lowest price the seller can accept without risk, and $h_{\text {low }}$ is the greatest price the buyer can afford to pay without risk. This interval has the following properties:

- Every price level outside the interval leads to an arbitrage opportunity.

- There are no arbitrage opportunities for price level in the interior of the interval.

The interval on pricing also implies an interval on hedging portfolios.

There is no one widely accepted method to calculate the incomplete market pricing interval.
The first approach is to find a super-replicating portfolio, see Cvitanic et al. ${ }^{2,3}$ This is a portfolio whose payoffs are always (in any state of the world) at least as big as the payoff of the options. The value of the options is then bounded by the value of the super-replicating portfolios. ${ }^{4}$ The second approach is utility-based valuation, which has been developed and studied by, among others, Hicks ${ }^{5}$ Hodges and Neuberger, Davis, Karatzas and Kou ${ }^{8}$ and Kallsen (2002). The investor of the contingent claims assumes unhedgable risk, which will affect the probability distribution of his consumption and final wealth level, and his utility. The certainty equivalent wealth of the expected utility is then the value an investor is prepared to pay for the claim. This wealth is the 'shadow' market value of the (partially) unhedgable claim. The final approach stems from a no-arbitrage argument, to compare different portfolio strategies involving the underlying asset and options, and work out the option bounds by excluding the existence of any dominant strategies.

Pricing bounds are useful in many situations in which a relative pricing approach is appropriate but perfect replication is not possible. A few examples follow: (1) A trader can use the bounds as buy and sell points in the search for 'good deals' in asset markets (with the usual warning question of why the market leaves good deals undiscovered). (2) A bank or other institution that markets or synthesises nontraded securities can use bounds as bid and ask prices.

(3) Bounds can be used as economic measures of the accuracy of option pricing formula. (4) Option pricing techniques are increasingly applied to 'real option' in capital budgeting, investment with irreversibility and policy questions. A relative pricing approach is appropriate but the focus payoffs typically cannot 
be perfectly replicated. (5) Option pricing formulas are often used in risk assessments to quantify the exposure of a position or institutions to various risk factors. It is useful to assess such risks when perfect replication is impossible and to quantify the importance of the market price of risk assumptions.

The paper is organised as follows. I start the second section with a description of the problem and methodology. The third and fourth sections show how to calculate lower and upper bounds on European option prices. The fifth section is meant to illustrate our results numerically and graphically. In the sixth section, I derive the corresponding options hedging intervals. The seventh section concludes.

\section{DESCRIPTION OF THE PROBLEM}

Consider a European call option on a risky asset with current price $S$ that matures at time $T$ having exercise prices $X$. In an arbitrage-free setting, the price of this option can be determined as

$$
C=\exp \left(-r_{f} T\right) E^{\mathrm{Q}}\left[\left(S_{T}-K\right)_{+}\right]
$$

where $r_{f}$ denotes the risk-free interest rate and where the stochastic process $\left\{S_{t}, t \geq 0\right\}$, starting at $S_{0}=S$, describes the price process of the underlying asset. I assume that $Q$ is the unique equivalent probability measure, such that the discounted price process is a martingale, or $E^{\mathrm{Q}}\left[\exp \left(r_{f} t\right) S_{t}\right]=S$.

Following Merton, ${ }^{10}$ the option price must satisfy

$$
\max \left[0, S-\exp \left(-r_{f} T\right) X\right] \leq C \leq S
$$

The Merton ${ }^{10}$ bounds require no knowledge of the underlying asset's terminal price distribution, which paved the way for subsequent research that produced increasingly tighter bounds by adding structure to the underlying asset's terminal price distribution and by further restricting investor behaviour.

A unique option price can only be obtained if the distribution of the underlying asset price process $\left\{S_{t}, t \geq 0\right\}$ is known for sure, which may not be a realistic assumption in some contexts. The most commonly used assumption is the Black and Scholes setting, where the price process is assumed to follow a geometric Brownian motion. This means that

$$
S_{t}=S\left[\exp \left(\left(r_{f}-\frac{1}{2} \sigma^{2}\right) t+\sigma W_{t}\right)\right]
$$

where $W_{t}$ is standard Brownian motion. Thus, under the measure $Q$ the variable $S_{t} / S$ are lognormally distributed with mean $r_{f}$ and variance $\sigma^{2} t$. Under these assumptions, the price of a European call can be found according to the well-known Black and Scholes formula

$$
C=S N\left(d_{1}\right)-X e^{-r_{f} T} N\left(d_{2}\right)
$$

with

$$
\begin{aligned}
& d_{1}=\frac{1}{\sqrt{\sigma^{2} T}}\left\{\ln (S / X)+\left(r_{f}+\frac{1}{2} \sigma^{2}\right) T\right\} \\
& d_{1}=\frac{1}{\sqrt{\sigma^{2} T}}\left\{\ln (S / X)+\left(r_{f}-\frac{1}{2} \sigma^{2}\right) T\right\}
\end{aligned}
$$

where $N\left(^{*}\right)$ denotes the cumulative distribution function of the standard normal distribution.

As mentioned in the Introduction, the Black and Scholes pricing formula shows some imperfections in that the whole distribution of the price process is fixed by means of a lognormal model. Although this model performs well in a lot of cases (it is still the most widely used approach for the valuation of options), wrong prices can arise due to strong assumptions. In order to avoid the problem of 
model risk, many approaches have been suggested to modify the assumptions.

Perrakis and Ryan ${ }^{11}$ improve the Merton ${ }^{10}$ bounds under very mild preference assumptions. While the bounds they develop require more information than the Black-Scholes models, they do not rely on the ability to create riskless hedges. Perrakis ${ }^{12}$ further tightens the lower bounds by restricting the range of allowable terminal underlying asset prices.

Levy $^{13}$ uses first- and second-order stochastic dominance concepts to derive option pricing bounds in a discrete setting. After obtaining Merton's ${ }^{10}$ bounds using first-order stochastic dominance, he tightens those bounds using second-order stochastic dominance analysis. In a simultaneous contribution, Richken ${ }^{14}$ uses linear programming to find the same bounds as Levy ${ }^{13}$ under additional assumptions on risk aversion and state probabilities. Practical limitations of the linear programming bounds are caused by the fact that, in addition to the volatility, the instantaneous mean return must be estimated.

Lo ${ }^{15}$ finds semi-parametric upper bounds with the property that only the first two moments of the underlying asset's terminal price distribution need to be known. Boyle and Lin ${ }^{16}$ extend Lo's results to options with multiple underlying assets. Laurence and Wang ${ }^{17}$ derive distribution-free sharp bounds for the price of a basket option.

Rodriguez $^{18}$ starts from a general expression for the call option prices as a sum of three components, one of which contains the discount factor of the corresponding put options. By focusing on this discount factor, he recognises the former results of Merton, Levy and others; he also extends the approach to a new tighter lower bound.

Schepper and Heijnen ${ }^{19}$ derive a new formula for option bounds by making use of the knowledge of successive moments of the real price distribution instead of the complete distribution in a risk-neutral setting.

\section{LOWER OPTION PRICING BOUND}

Rodriguez ${ }^{18}$ presents a single framework to derive various previous traditional lower pricing bounds. Assuming $C$ and $P$ are the current price of a European call and put option respectively on the same underlying asset, with the exercise price $X$ and time to maturity $T$. Current asset price is $S$ and its terminal price when the options expire is $S_{T}$, with cumulative distribution function $F\left(S_{T}\right)$. The risk-free rate is $r_{f}$, and the required rate of return on the underlying asset, call and put are $r_{S}, r_{C}$ and $r_{P}$, respectively.

The terminal price of a call option is $C_{T}=\max \left[0, S_{T}-\mathrm{X}\right]$; its expected value is found by integrating $C_{T}$ over $S_{T} \in[0, \infty)$ and simplifying

$$
\begin{aligned}
E\left(C_{T}\right)= & \int_{X}^{\infty} S_{T} \mathrm{~d} F\left(S_{T}\right)-X[1-F(X)] \\
E\left(C_{T}\right)= & \int_{0}^{\infty} S_{T} \mathrm{~d} F\left(S_{T}\right)-\int_{0}^{X} S_{T} \mathrm{~d} F\left(S_{T}\right) \\
& -X[1-F(X)]
\end{aligned}
$$

where $\int_{0}^{\infty} S_{T} \mathrm{~d} F\left(S_{T}\right)$ is the expected underlying asset price at the option's expiration, which can be written as $E\left(S_{T}\right)=S^{*} \exp \left(r_{S} T\right)$. Also, using the product rule of calculus on the second integral in the above equation yields the general expression of the expected terminal price of a call European option:

$$
E\left(C_{T}\right)=S e^{r_{S} T}-X+\int_{0}^{X} F\left(S_{T}\right) \mathrm{d}\left(S_{T}\right)
$$


To find the current price of a call option $C(S)$, each component in the right-hand side of equation (5) should be discounted at a rate commensurate with its risk. The first term in the right-hand side is the expected value of the underlying asset's terminal price, which should be discounted at its required rate, $r_{s}$. The second term is the exercise price, and because it is known with certainty, it must be discounted at the risk free rate, $r_{f}$. And the integral term in equation (5) is the expected value of the put option at maturity, which should be discounted at the put's appropriate rate, $r_{P}{ }^{18,20}$ To simplify notation, let the integral part in equation (1) be denoted by $I(S)$ and let $R_{i} \equiv \exp \left(-r_{i} T\right)$ for $i=f, S, C, P$ represent the discount factors. Rodriguez ${ }^{18}$ presents the general expression for the current price of a European call option:

$$
C(S)=S-X R_{f}+R p(S) I(S)
$$

In fact, Merton's ${ }^{10}$ lower pricing bound, $C \geq \max \left[0, S-X^{*} R_{f}\right]$, can be obtained by assuming $R_{P}(S)$ in equation (2) is 0 . Hence, the functional form of $R_{P}(S)$ is

$$
R_{P}(S)=\frac{C(S)-S+X R_{f}}{I(S)}=V(S)+\frac{C(S)}{I(S)}
$$

where

$$
V(S)=\frac{X R_{f}-S}{I(S)}
$$

Because $V(S)$ always lies below $R_{P}(S)$, this implies that if some function $V_{k}(S)$ such that $V(S) \leq V_{k}(S) \leq R_{P}(S)$ is substituted for $R_{P}(S)$ in equation (2), the following lower bound function is obtained:

$$
L(S)=\max \left[0, S-X R_{f}+V_{k}(S) I(S)\right]
$$

All traditional lower bounds mentioned in the induction section can be explained by equation (8) by choosing different constant $V_{k}(S)$ function, and Rodriguez ${ }^{18}$ suggests a new tighter bound by expanding the set of allowable $V_{k}(S)$ beyond the class of constant functions. To illustrate the synthesising power of equation (8), several traditional lower bounds are reproduced in Rodriguez.

Merton's ${ }^{10}$ well-known lower bound is as follows:

$$
L_{M}(S)=\max \left[0, S-X R_{f}\right]
$$

Perrakis and Ryan's

$$
L_{P R}(S)=\max \left[0, S-X R_{f}+R_{f} I(S)\right]
$$

Levy's ${ }^{13}$ and Richken's ${ }^{14,21}$

$$
\begin{aligned}
L_{L / R}(S)= & 0 \quad \text { where } 0 \leq S<S_{\max } \\
= & S-X R_{f}+V\left(S_{\max }\right) I(S) \\
& \text { where } S \geq S_{\max }
\end{aligned}
$$

Rodriguez's ${ }^{18}$ new lower pricing bound, where $V^{\prime}\left(S_{i}\right)$ is the slope of inflexion line:

$$
\begin{aligned}
L_{R}(S)= & \max \left[0, S-X R_{f}\right. \\
& \left.+\left\{V\left(S_{i}\right)+V^{\prime}\left(S_{i}\right)\left(S-S_{i}\right)\right\} I(S)\right]
\end{aligned}
$$

The preceding discussion shows that $L_{M}(S) \leq L_{P R}(S) \leq L_{L / R}(S) \leq L_{R}(S) \leq C(S)$.

European call and put option with the same strike price, expiration date and underlying asset are not independent. In fact, any one of them can be replicated by the other two securities: a put option is a portfolio of the stock and the call options. This relationship is called the put-call parity, and was first proposed by Stoll. ${ }^{22}$ Thereby, the lower bound of a put option can be 
determined from the call lower bound using the put-call parity relation $P=C-S+X R_{f}$.

\section{UPPER OPTION PRICING BOUND}

Perrakis and Ryan, ${ }^{11}$ Richken, ${ }^{14}$ Levy ${ }^{13}$ and Rodriguez ${ }^{18}$ develop parametric option-pricing upper bounds, where the particular values of the bounds depend critically on the specific stock price distribution assumed. To find an upper bound, recall $C(S)=\exp \left(-r_{C} T\right)^{*} E\left(C_{T}\right)$, suggested in equation (5). Because $C_{T}=\max$ $\left[0, S_{T}-X\right]$, the cumulative distribution function of $C_{T}, H\left(C_{T}\right)$ and of $S_{T}, F\left(S_{T}\right)$ obey the relation $H(w)=F(X+w)$ for $w \geq 0$. This implies that $S_{T}$ stochastically dominates $C_{T}$ in the first order sense, so $C_{T}$ is riskier than $S_{T}$ in the Rothschild and Stiglitz ${ }^{23}$ sense. As a result, risk-averse investors require a higher rate of return on the call option than on its underlying asset, that is, $r_{C}>r_{S}{ }^{23,24}$ So $C(S)=\exp \left(-r_{C} T\right)^{*} E\left(C_{T}\right)<\exp$ $\left(-r_{S} T\right) * E\left(C_{T}\right)$. Substituting this in equation (5), the upper bound for the price of a call option is

$$
U(S)=S-X R_{S}+R_{S} I(S)
$$

Lo ${ }^{15}$ derives upper bounds for option prices and for expected pay-offs that depend no longer on the distribution but on the mean and variance of the terminal asset prices (two orders of terminal asset prices). Corresponding semi-parametric upper bounds can be derived for option prices when the variance of the associated risk-neutral pricing distribution is known. Lo's ${ }^{15}$ upper bounds for call and put options are

$$
U_{L}=\frac{S-X R_{f}+S V^{*} R_{f}^{2}}{1+V^{*} R_{f}^{2}}
$$

if

$$
\begin{gathered}
\frac{S}{X} \geq \frac{2 R_{f}}{1+V^{*} R_{f}^{2}} \\
=\frac{1}{2}\left[S-X R_{f}+\sqrt{\left(X R_{f}-S\right)^{2}+S^{2} V^{*} R_{f}^{2}}\right]
\end{gathered}
$$

if

$$
\frac{S}{X}<\frac{2 R_{f}}{1+V^{*} R_{f}^{2}}
$$

where

$$
V^{*}=R_{f}^{2}\left[\exp \left(\sigma^{2} T-1\right)\right]
$$

$V^{*}$ and $\sigma^{2}$ are the risk-neutral variance and underlying asset variance, respectively, assuming that underlying asset is lognormally distributed.

Put upper bound can be derived directly from the call upper bound using the put-call parity relation $\bar{P}=\bar{C}-S+X R_{f}$. It can readily be shown that these bounds satisfy many wellknown properties of rationally determined option prices, for example increasing in $r_{f}$, $T$, and $V^{*}$ and homogeneity of degree one in $S$ and $X$.

\section{CALIBRATION}

In this the first section calibrates the option pricing bounds method with an illustrative sample. The underlying asset's price return, $S_{T} / S$ is assumed lognormally distributed with parameters $\mu$ and $\sigma^{2}$, so that $r_{S}=\mu+\sigma^{2} / 2$ and $I(S)$ is

$$
I(S)=X N\left(-a_{2}\right)-\frac{S}{R_{S}} N\left(-a_{1}\right)
$$

where $N\left(^{*}\right)$ represents the standard normal cumulative distribution function and ${ }^{25}$

$$
a_{1}=\frac{\ln (S / X)+\left(r_{s}+\sigma^{2} / 2\right) T}{\sigma \sqrt{T}}
$$

$$
a_{2}=a_{1}-\sigma \sqrt{T}
$$




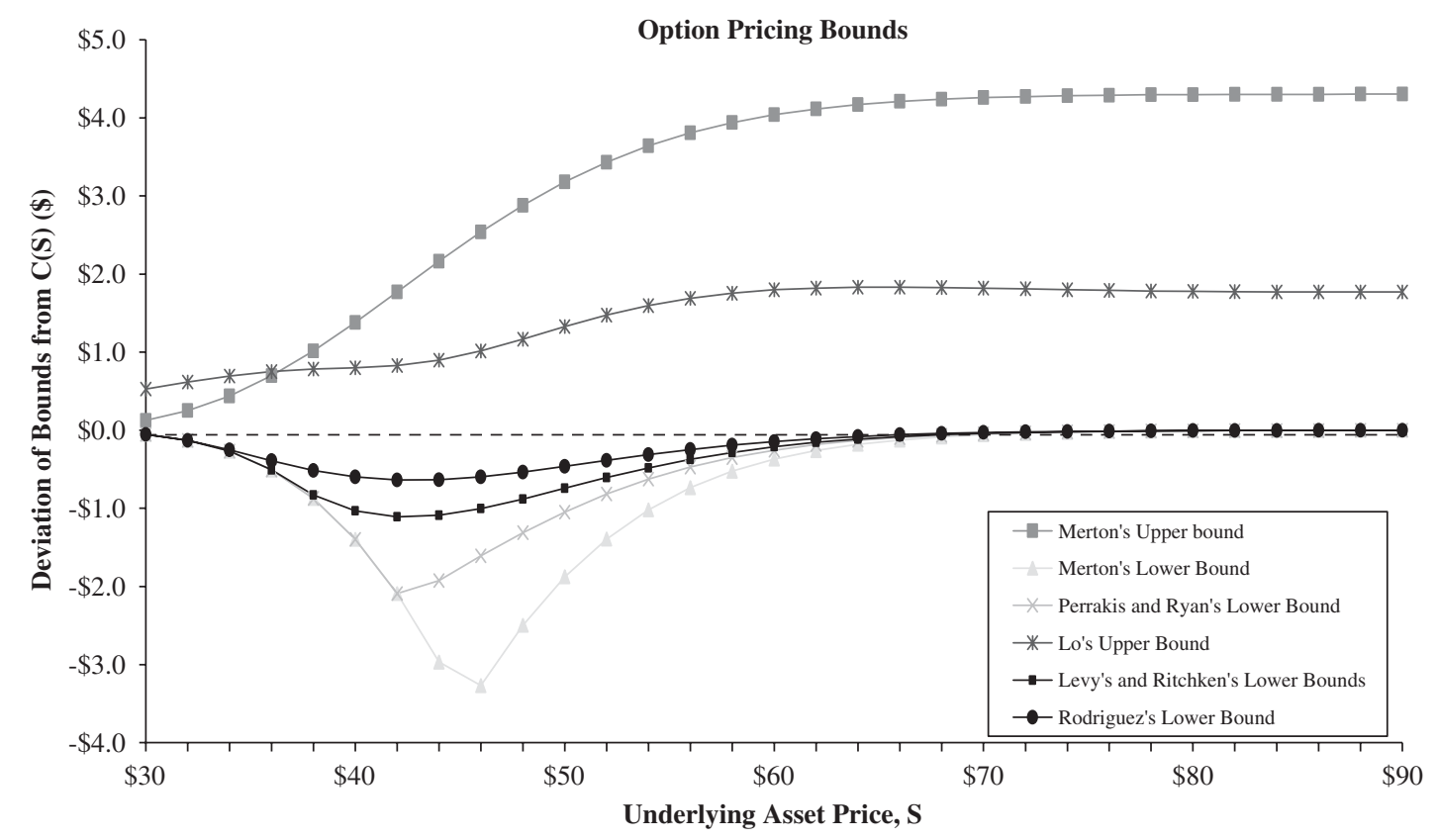

Figure 1: Deviation of lower and upper pricing bounds from the Black-Scholes call price, $\$\left(X=\$ 50, T=1\right.$ year, $\left.r_{f}=0.1, \mu=0.18, \sigma=0.20\right)$

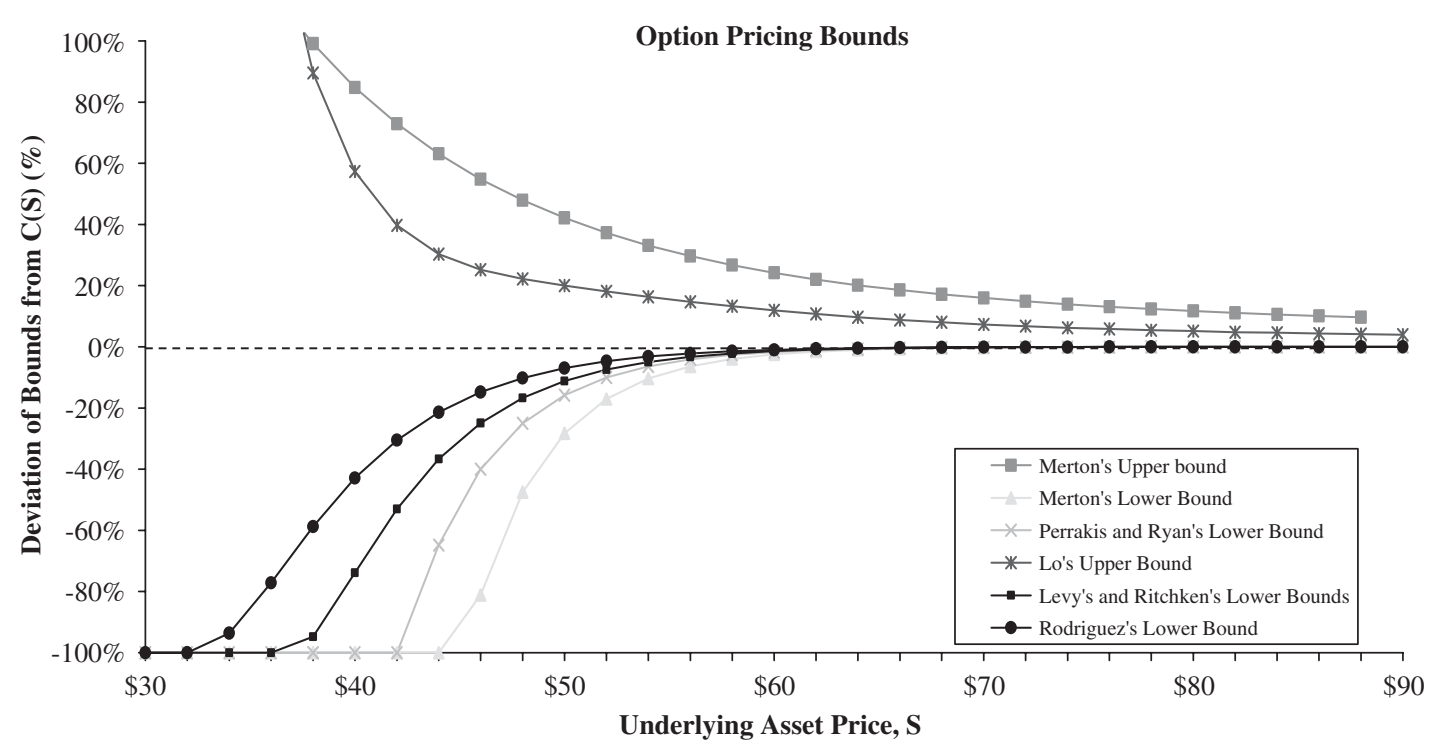

Figure 2: Deviation of lower and upper pricing bounds from the Black-Scholes call price, $\%\left(X=\$ 50, T=1\right.$ year, $\left.r_{f}=0.1, \mu=0.18, \sigma=0.20\right)$ 
All the lower and upper option pricing bounds discussed in previous sections are calculated, employing the same assumption used in Rodriguez $^{18}$ parameters $X=\$ 50, T=1$ year, $r_{f}=0.1, \mu=0.18, \sigma=0.2$ and implied $r_{s}=0.2$. Table 1 summarises results of various bounds and Black-Scholes price.
Figures 1 and 2 graph the deviation of each pricing bound from the Black and Scholes price in real value and percentage terms. Both figures confirm that the deviations of lower bounds are progressively tighter when asset price $(S)$ increases, and deviations of upper bounds increase toward a constant level gradually with $S$, although

Table 1: Lower and upper bounds for call options price

\begin{tabular}{lccccccc}
\hline$S$ & $L_{M}(S)$ & $L_{P R}(S)$ & $L_{L / R}(S)$ & $L_{R}(S)$ & $C_{B S}(S)$ & $U_{L o}(S)$ & $U_{M}(S)$ \\
\hline$\$ 30$ & $\$ 0.000$ & $\$ 0.000$ & $\$ 0.000$ & $\$ 0.000$ & $\$ 0.054$ & $\$ 0.580$ & $\$ 0.181$ \\
$\$ 32$ & $\$ 0.000$ & $\$ 0.000$ & $\$ 0.000$ & $\$ 0.000$ & $\$ 0.128$ & $\$ 0.747$ & $\$ 0.379$ \\
$\$ 34$ & $\$ 0.000$ & $\$ 0.000$ & $\$ 0.000$ & $\$ 0.017$ & $\$ 0.269$ & $\$ 0.966$ & $\$ 0.709$ \\
$\$ 36$ & $\$ 0.000$ & $\$ 0.000$ & $\$ 0.000$ & $\$ 0.116$ & $\$ 0.507$ & $\$ 1.259$ & $\$ 1.204$ \\
$\$ 38$ & $\$ 0.000$ & $\$ 0.000$ & $\$ 0.045$ & $\$ 0.360$ & $\$ 0.873$ & $\$ 1.656$ & $\$ 1.890$ \\
$\$ 40$ & $\$ 0.000$ & $\$ 0.000$ & $\$ 0.365$ & $\$ 0.797$ & $\$ 1.395$ & $\$ 2.195$ & $\$ 2.777$ \\
$\$ 42$ & $\$ 0.000$ & $\$ 0.000$ & $\$ 0.983$ & $\$ 1.452$ & $\$ 2.090$ & $\$ 2.921$ & $\$ 3.862$ \\
$\$ 44$ & $\$ 0.000$ & $\$ 1.044$ & $\$ 1.881$ & $\$ 2.333$ & $\$ 2.968$ & $\$ 3.867$ & $\$ 5.132$ \\
$\$ 46$ & $\$ 0.758$ & $\$ 2.416$ & $\$ 3.024$ & $\$ 3.429$ & $\$ 4.026$ & $\$ 5.041$ & $\$ 6.564$ \\
$\$ 48$ & $\$ 2.758$ & $\$ 3.941$ & $\$ 4.374$ & $\$ 4.718$ & $\$ 5.253$ & $\$ 6.420$ & $\$ 8.134$ \\
$\$ 50$ & $\$ 4.758$ & $\$ 5.589$ & $\$ 5.893$ & $\$ 6.172$ & $\$ 6.635$ & $\$ 7.962$ & $\$ 9.815$ \\
$\$ 52$ & $\$ 6.758$ & $\$ 7.333$ & $\$ 7.543$ & $\$ 7.764$ & $\$ 8.149$ & $\$ 9.625$ & $\$ 11.584$ \\
$\$ 54$ & $\$ 8.758$ & $\$ 9.151$ & $\$ 9.295$ & $\$ 9.463$ & $\$ 9.776$ & $\$ 11.374$ & $\$ 13.419$ \\
$\$ 56$ & $\$ 10.758$ & $\$ 11.023$ & $\$ 11.120$ & $\$ 11.246$ & $\$ 11.493$ & $\$ 13.185$ & $\$ 15.303$ \\
$\$ 58$ & $\$ 12.758$ & $\$ 12.935$ & $\$ 13.000$ & $\$ 13.092$ & $\$ 13.283$ & $\$ 15.040$ & $\$ 17.224$ \\
$\$ 60$ & $\$ 14.758$ & $\$ 14.875$ & $\$ 14.918$ & $\$ 14.985$ & $\$ 15.129$ & $\$ 16.928$ & $\$ 19.169$ \\
$\$ 62$ & $\$ 16.758$ & $\$ 16.835$ & $\$ 16.863$ & $\$ 16.910$ & $\$ 17.018$ & $\$ 18.840$ & $\$ 21.133$ \\
$\$ 64$ & $\$ 18.758$ & $\$ 18.808$ & $\$ 18.826$ & $\$ 18.859$ & $\$ 18.938$ & $\$ 20.770$ & $\$ 23.109$ \\
$\$ 66$ & $\$ 20.758$ & $\$ 20.790$ & $\$ 20.802$ & $\$ 20.825$ & $\$ 20.882$ & $\$ 22.715$ & $\$ 25.093$ \\
$\$ 68$ & $\$ 22.758$ & $\$ 22.779$ & $\$ 22.786$ & $\$ 22.802$ & $\$ 22.843$ & $\$ 24.670$ & $\$ 27.082$ \\
$\$ 70$ & $\$ 24.758$ & $\$ 24.771$ & $\$ 24.776$ & $\$ 24.787$ & $\$ 24.816$ & $\$ 26.635$ & $\$ 29.075$ \\
\hline & & & & & & & \\
& & & &
\end{tabular}

Note: All calculations assume a lognormal distribution for $S_{T}$, with parameters $X=\$ 50, T=1$ year, $r_{f}=0.1, \mu=0.18$ and $\sigma=0.20$. Four lower bounds, two upper bounds and Black-Scholes option price are shown in columns 2 through 8 .

$L_{M}(S)=$ Merton's ${ }^{10}$ lower bound for the call option price; $L_{P R}(S)=$ Perrakis and Ryan's ${ }^{11}$ lower bound; $\mathrm{L}_{\mathrm{L}}$ / ${ }_{\mathrm{R}}(S)=$ Levy's ${ }^{13}$ and Richken's ${ }^{14}$ lower bound; $L_{R}(S)=$ Rodriguez's 'lower bound; $C_{B S}(S)=$ call options price according to the Black and Scholes ${ }^{1}$ model; $U_{L o}(S)=$ Lo's upper bound; $U_{M}(S)=$ Merton's ${ }^{10}$ upper bound. 
in term of percentage both upper and lower bounds converge to the Black and Scholes price.

The width of the bounds is larger at-themoney than it is in-the-money or out-of-themoney. Options are hardest to hedge at the money because the nonlinearity of the option payoff as a function of stock price is greatest here. Therefore, the residual - option payoff less best bounds - is largest in this region. The width of the bounds, however, is a much larger fraction of the call option value for out-of-themoney options on the left-hand side of the chart. In this sense, as well as when translated to implied volatilities, the bounds are wider for out-of-the-money options.

\section{UPPER AND LOWER DELTA HEDGING BOUNDS}

Option pricing theory is used extensively to quantify risk exposure by measuring how much an option value would change if an underlying asset price changed. This sensitivity is known as the option's delta. The delta of a call is differentiation (4) with respect to $S$, and the result is

$$
\Delta_{\text {call }}(S, X, T)=\frac{\partial}{\partial S} C(S, X, T)=N\left(d_{1}\right)
$$

The delta of a put is obtained immediately from put-call parity, which equals $N\left(d_{1}\right)-1$.

Assume that the delta of the 'at-the-money' call option discussed in Table 1 is 0.6. This means that when the stock price changes by a small amount, the option price changes by approximately 60 per cent of that amount. Suppose the option price is $\$ 10$ and the stock price is $\$ 100$. An investor who has sold 20 call options contract, that is, options to buy 2,000 shares, the investor's position could be hedged by buying $0.6 * 2,000=1,200$ shares. The gain (loss) on the options positions would tend to be offset by the loss (gain) on the stock position. For example, if the stock price goes up by $\$ 1$ (producing a gain of $\$ 1,200$ on the shares purchased), the option price will tend to go up by $\$ 1 * 0.6=\$ 0.6$ (producing a loss of $\$ 1,200$ on the options written). In this example, the delta of the investor's option position is 0.6 $*(-2,000)=-1,200$. Hence, the delta of the total position (short 2,000 call options and long 1,200 shares) is zero. The delta of the position in the underlying asset offsets the delta of the option position. A position with a delta of zero is referred to as being delta neutral.

It is important to realise that the investor's position remains delta hedged for only a relatively short period of time $(\Delta t)$. This is because delta changes with both changes in the stock price and the passage of time. In practice, when delta hedging is implemented, the hedge has to be adjusted or 'rebalanced' periodically.

The existence of an 'arbitrage-free' interval, instead of a single Black-Scholes price, suggests that the delta hedging ratio is not unique. The delta hedging ratio corresponding to lower and upper bounds presented in the third and fourth sections are calculated below.

The following are the delta hedging ratios from the various studies reviewed in this paper:

- Merton's ${ }^{10}$ lower-bound

$$
\Delta_{M}(S)=1
$$

Perrakis and Ryan's

$$
\left.\Delta_{P R}(S)=1+R_{f} I^{\prime}(S)\right]
$$


Delta hedging bounds

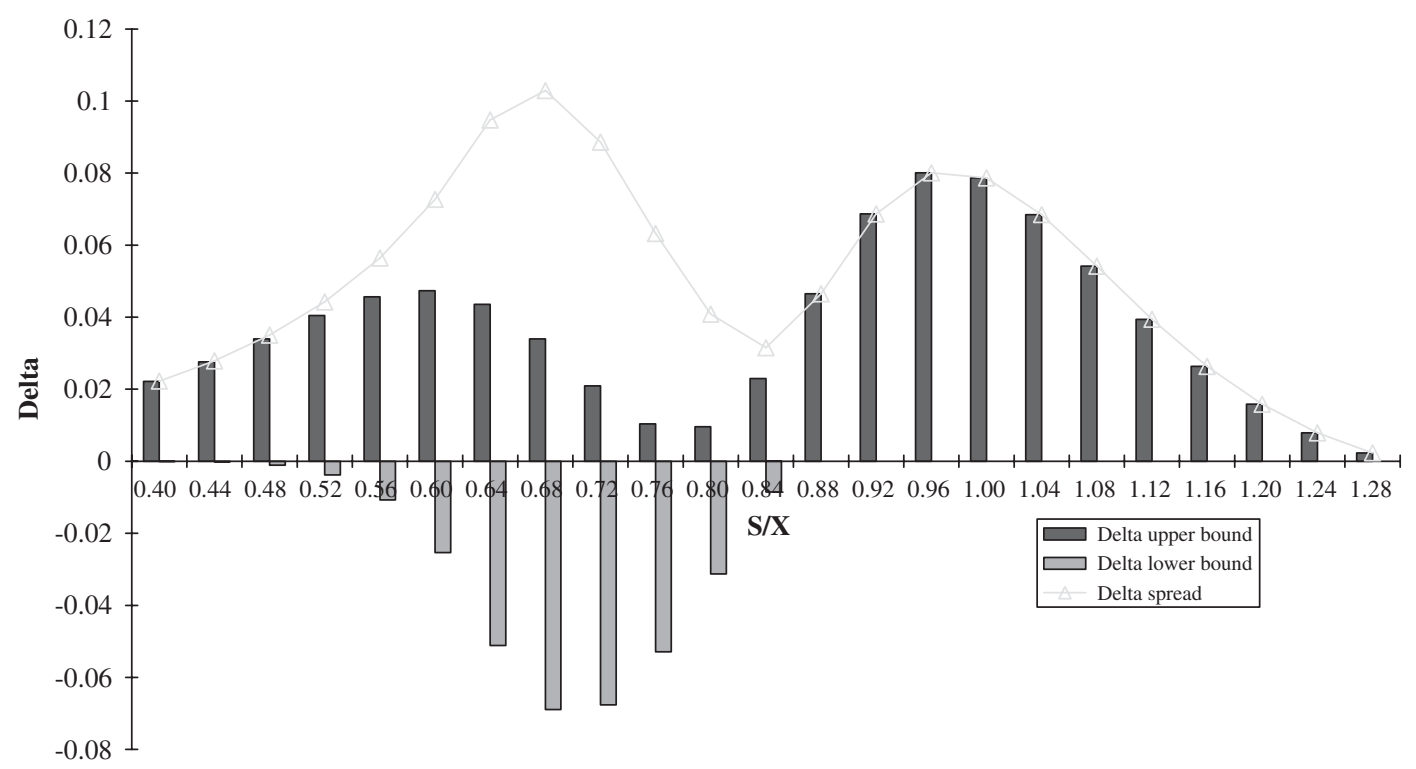

Figure 3: Delta hedging bounds

Levy's ${ }^{13}$ and Richken's ${ }^{14}$

$$
\Delta_{L / R}(S)=1+V\left(S_{\max }\right) I^{\prime}(S)
$$

where $\mathrm{S} \geq \mathrm{S}_{\max }$

Rodriguez's ${ }^{18}$ new lower pricing bound is

$$
\begin{aligned}
\Delta_{R}(S)= & 1+\left[V\left(S_{i}\right)-V^{\prime}\left(S_{i}\right) S_{i}\right] I^{\prime}(S) \\
& +V^{\prime}\left(S_{i}\right) I(S) \\
& +V^{\prime}\left(S_{i}\right) S I^{\prime}(S)
\end{aligned}
$$

where

$$
I^{\prime}(S)=-N\left(-a_{1}\right) / R_{S}
$$

Lo's $^{15}$ upper bounds are

$$
\Delta_{L}=1
$$

if

$$
\frac{S}{X} \geq \frac{2 R_{f}}{1+V^{*} R_{f}^{2}}
$$

$$
=\frac{1}{2}\left[1+\frac{-2\left(R_{f} X-S\right)+2 R_{f}^{2} V^{*} S}{2 \sqrt{\left(R_{f} X-S\right)^{2}+R_{f}^{2} V^{*} S^{2}}}\right]
$$

if

$$
\frac{S}{X}<\frac{2 R_{f}}{1+V^{*} R_{f}^{2}}
$$

where

$$
V^{*}=R_{f}^{2}\left[\exp \left(\sigma^{2} T-1\right)\right]
$$

Figure 3 shows hedging bound of corresponding Lo's upper ${ }^{15}$ and Rodriguez's lower price bounds ${ }^{18}$ over Black delta. The lower 


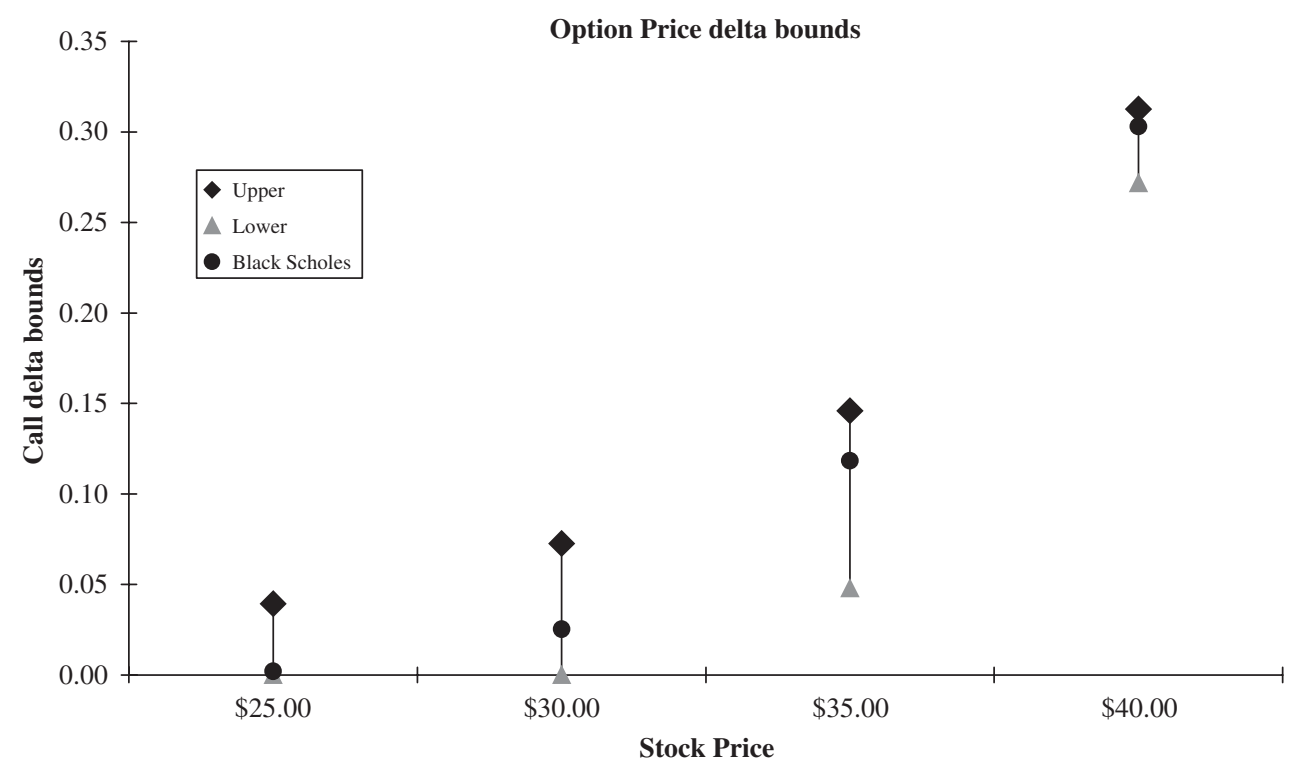

Figure 4: Option price delta bound

hedging bound is much tighter than the upper bound. The lower hedging bounds converge towards Black delta for the ratio of underlying asset price over the strike price is less than about 0.5 or greater than 0.9 . The width of the hedging bounds follows the similar trends as price bounds, and is larger for close-to-themoney options.

Figure 4 presents a graph of upper and lower delta bounds for the out-of-money option when the stock price ranges from $\$ 25$ to $\$ 40$. Because the slope of Rodriguez's lower price bounds converges to Black-Scholes prices faster than Lo's upper price bounds, Black delta moves away from lower bound towards upper bound as price rises. In this numerical example, the delta bounds widen when stock price rise to $\$ 35$ and narrows afterwards.

In sum, delta hedging bounds can be used in this way to quantify risk exposure and to assess the uncertainty in risk exposure calculations and hedging portfolios design. A number of factors, however, may change the conclusions. Higher order risks, that is, gamma and theta, are not considered in proposed delta hedging, which may affect the hedging portfolio interval.

\section{CONCLUSIONS}

This paper has reviewed the literature on options pricing in incomplete markets. Tight upper and lower bounds can be derived based on the assumptions of mean and variance of the underlying asset price, not on its entire distribution. The differences between estimated upper or lower bounds and Black-Scholes price are quite small for deep in-the-money options, but can be very significant for deep out-of-themoney options. But at the same time, despite the wide pricing bounds, analysis of the implied hedging bounds suggests that the implications for asset allocation of incomplete markets are fairly limited. The most important assumption behind these results is the time invariance of the 
parameters, that is means and variances.

Nonstationarity in the underlying parameters may well change these conclusions.

\section{Acknowledgments}

I thank Michael Orszag for comments and suggestions on the paper.

\section{References and Notes}

1 Fischer, B. and Myron, S. (1973) 'The Pricing of Options and Corporate Liabilities', Journal of Political Economy, Vol. 81, No. 3, pp. 637-654.

2 Cvitanic, J., Pham, H. and Touzi, N. (1998) 'A Closed form Solution to the Problem of Super-Replication Under Transaction Costs', Finance and Stochastic, Vol. 3, pp. 35-54.

3 Cvitanic, J., Pham, H. and Touzi, N. (1999) 'SuperReplication in Stochastic Volatility Models Under Portfolio Constraints', Journal of Applied Probability, Vol. 36, pp. 523-545.

4 This approach runs into problems, particularly where there are unbounded variables and therefore no superreplicating portfolios.

5 Hicks, J.R. (1956) 'A Revision of Demand Theory', Oxford University Press, London.

6 Hodges, S.D. and Neuberger, A. (1989) 'Optimal Replication of Contingent Claims Under Transaction Costs', Rev. Futures Mkts, Vol. 8, pp. 222-239.

7 Davis, M.H.A. (1997) 'Options Pricing in Incomplete Market', in Dempster, M. A. H. and Pliska, S. R. (eds.) 'Mathematics of Derivatives Securities', Cambridge University Press, New York, pp. 216-226.

8 Karatzas, I. and Kou, S.G. (1996) 'On the Pricing of Contingent Claims Under Constraints', Ann. Appl. Prob, Vol. 6, No. 2, pp. 321-369.

9 Kallsen, J. (2002) 'Derivative Pricing Based on Local Utility Maximization', Finance Stoch., Vol. 6, pp. 115-140.

10 Merton, R.C. (1973) 'Theory of Rational option Pricing', Bell Journal of Economics and Management Science, Vol. 4, pp. 141-183.

11 Perrakis, S. and Ryan, P.J. (1984) 'Option Pricing Bounds in Discrete Time', Journal of Finance, Vol. 39, pp. 519-525.
12 Perrakis, S. (1986) 'A Discrete Time Option Model Dependent on Expected Return: A Note', Journal of Business, Vol. 59, pp. 119-142.

13 Levy, H. (1985) 'Upper and Lower Bounds of Put and Call Option Value: Stochastic Dominance Approach', The Journal of Finance, Vol. 60, No. 4, pp. 1197-1217.

14 Richken, P. (1985) 'On Options Pricing Bounds', Journal of Finance, Vol. 40, pp. 1219-1233.

15 Lo, A.W. (1987) 'Semi-Parametric Upper Bounds for Option Prices and Expected Payoffs', Journal of Financial Economics, Vol. 19, pp. 373-387.

16 Boyle, P.P. and Lin, X.S. (1997) 'Bounds on Contingent Claims Based on Several Assets', Journal of Financial Economics, Vol. 46, pp. 383-400.

17 Laurence, P. and Wang, T.-H. (2005) 'Sharp Upper and Lower Bounds for Basket options', Applied Mathematical Finance, Vol. 12, No. 3, pp. 253-282.

18 Rodriguez, R.J. (2003) 'Option Pricing Bounds: Synthesis and Extension', Journal of Financial Research, Vol. XXVI, No. 2, pp. 149-164.

19 Schepper, A.D. and Heijnen, B. (2007) 'Distributionfree Option Pricing', Insurance: Mathematics and Economics, Vol. 40, pp. 179-199.

20 Rodriguez $(2003)^{18}$ notes that $R_{f}$ and $R_{S}$ are constant in the model, but $R_{C}$ and $R_{P}$ depend on the value of the underlying asset price, $S$. To gain intuition on this dependence, note that a very deep in-themoney call option is almost sure to have a terminal payoff close to $S_{T}$. This means $R c$ asymptotically approaches $R_{S}$ as $S$ increase. Similarly, $R_{P}$ approaches $R_{f}$ as $S$ get closer to zero. Both limits indicate that $R_{P}$ and $R_{C}$ depend on $S$.

21 The $V(S)$ function starts at the point $\left(0, R_{f}\right)$, then increases monotonically with $S$, reaching a unique maximum at $\left(S_{\max }, V\left(S_{\max }\right)\right)$, then decreases monotonically, passing through the point $\left(X R_{f}, 0\right)$.

22 Stoll, H.R. (1969) 'The Relationship Between Put and Call Option Price', Journal of Finance, Vol. 31, pp. 319-332.

23 Rothschild, M. and Stiglitiz, J.E. (1970) 'Increasing Risk I: A Definition', Journal of Economic Theory, Vol. 2, pp. 225-243.

24 This result is theorem 8 in Merton (1973) and relies critically on the Rothschild and Stiglitz $(1970)^{23}$ definition of risk.

25 Equation 7 is a Black-Scholes formula for the price of a European put option, except that each term $r_{S}$ is used instead of $r_{f}$. 